\title{
Non-Abelian spin-orbit gauge: Persistent spin helix and quantum square ring
}

\author{
Son-Hsien Chen* and Ching-Ray Chang \\ Department of Physics, National Taiwan University, Taipei 10617, Taiwan \\ (Received 31 August 2007; published 22 January 2008)
}

\begin{abstract}
We reexpress the Rashba and Dresselhaus interactions as non-Abelian spin-orbit gauges and provide a perspective in understanding the persistent spin helix [B. A. Berneving et al., Phys. Rev. Lett. 97, 236601 (2006)]. A spin-orbit interacting system can be transformed into a free-electron gas in the equal-strength Rashba-Dresselhaus [001] linear model, the Dresselhaus [110] linear model, and a one-dimensional system. A general tight-binding Hamiltonian for nonuniform spin-orbit interactions and hoppings along arbitrary directions, within the framework of finite-difference method, is obtained. As an application based on this Hamiltonian, a quantum square ring in contact with two ideal leads is found to exhibit four states: insulating, spin-filtering, spin-flipping, and spin-keeping states.
\end{abstract}

DOI: 10.1103/PhysRevB.77.045324

PACS number(s): 72.25.Dc, 85.75.-d, 73.21.Hb, 03.65.Vf

\section{INTRODUCTION}

The spin-orbit (SO) interaction interests scientists not only for its wide applications in spintronics devices but also for its profound physical significance. As frequently discussed, the Rashba spin-orbit (RSO) interaction and the Dresselhaus spin-orbit (DSO) interaction exist in twodimensional electron gas (2DEG) which exists in semiconductor heterostructures. The former, with strength adjustable via the gate voltage, ${ }^{1,2}$ is due to the inversion asymmetry of the structure, ${ }^{3}$ while the latter is due to lack of bulk inversion symmetry. ${ }^{4,5}$ The SO coupling acts as an effective momentum-dependent magnetic field ${ }^{6}$ and thus rotates the spin. The precession angle acquired by an electron varies according to the actual travel path of the electron. However, in the two special cases of Rashba-Dresselhaus [001] (linear) model of equal-strength RSO and DSO couplings and of the Dresselhaus [110] (linear) model, this angle exhibits unique behavior in that it is determined merely by the traveling distance along a specific spatial direction. In other words, the spin is rotated by an identical angle for all paths with the same projection to this direction. It turns out that the configuration or the spatial distribution of the spin polarizations is depicted by a helixlike pattern. Additionally, the pattern is persistent under any spin-independent scattering; this persistency implies that the spin lifetime is infinite and thus represents the so-called persistent spin helix ${ }^{7,8}$ (PSH). The utilization of this persistency was proposed by Schliemann et al. ${ }^{9}$ to achieve a spin-field-effect transistor with no ballistic condition required.

Earlier study by Hatano et al. ${ }^{10}$ showed that SO interactions can be regarded as non-Abelian $\mathrm{SO}$ or $S U(2)$ gauges, $\mathbf{A}^{\mathrm{SO}}=\left(A_{x}^{\mathrm{SO}}, A_{y}^{\mathrm{SO}}, 0\right)$, which impose spin-dependent phases on the traveling electron. By neglecting the DSO coupling and further adjusting the strengths of the RSO coupling and the magnetic field, they achieved a perfect spin-filtering ring in which one spin component gains destructive interference while the other gains constructive one. Nonetheless, the more realistic case of the coexistence of RSO and DSO interactions has not yet been considered.

In this paper, we consider the RSO and DSO interacting systems subject to an external magnetic field. We point out that, even with the coexistence of RSO and DSO couplings, the SO interactions and the magnetic field can all be regarded as gauges. To obtain a discrete (in space) tightbinding (TB) model, we employ the finite-difference ${ }^{11}$ (FD) method and introduce the $S U(2)$ gauge with similar procedure used for $U(1)$ gauge. The conditions for performing this analogy will be specified. We justify this SO-interacting TB model by checking its consistency with a previously proposed one. ${ }^{12}$ Utilizing these gauges, we show first that for a continuous Hamiltonian, the predicted $\mathrm{PSH}^{7,8}$ can be understood easily from the perspective of gauge transformation, and that for a discrete TB Hamiltonian, a square ring functions as a versatile device with four states: insulating, spin filtering, ${ }^{10}$ spin flipping, and spin keeping.

This paper is organized as follows. The two sections, II and III are divided into two parts which focus, respectively, on the continuous and discrete cases. The Hamiltonian is studied in the continuous case in Sec. II A and in the discrete case in Sec. II B. Applications for both cases are given in Secs. III A and III B. The Rashba-Dresselhaus [001] linear Hamiltonian is considered throughout this paper, except in Sec. III B where we address also the Dresselhaus [110] linear model to demonstrate the PSH. We summarize in Sec. IV.

\section{SPIN-ORBIT GAUGE IN THE RASHBA-DRESSELHAUS [001] LINEAR HAMILTONIAN}

In this section, we study the single-particle RashbaDresselhaus [001] linear Hamiltonian. In Sec. II A, we introduce the RSO and DSO interactions and show that they can be expressed as gauges. To make this Hamiltonian numerically treatable, we discretize it in Sec. II B by considering a TB model for the free-electron system subject to an external magnetic field.

\section{A. Continuous case}

Spin-orbit interactions in 2DEG (in the $x-y$ plane) are commonly modeled by taking into account the lowest order (linear) in momentum p. Consider a heterostructure grown along the [001] direction. The corresponding single-particle 
Hamiltonian, together with the kinetic energy and the magnetic gauge $\mathbf{A}^{B}$, reads

$$
H=\frac{\Pi^{2}}{2 m}+\frac{\alpha}{\hbar}\left(\Pi_{y} \sigma_{x}-\Pi_{x} \sigma_{y}\right)+\frac{\beta}{\hbar}\left(\Pi_{x} \sigma_{x}-\Pi_{y} \sigma_{y}\right),
$$

where $\alpha$ and $\beta$ denoting the RSO and DSO coupling strengths. The magnetic field $\mathbf{B}$ is introduced by the kinetic momentum $\boldsymbol{\Pi}=\mathbf{p}-e \mathbf{A}^{B} / c$. Since Eq. (1) is quadratic in $\boldsymbol{\Pi}$, one can always shift the operator $\mathbf{I}$ so that the linear term disappears. Introducing the SO gauge,

$$
\mathbf{A}^{\mathrm{SO}}=\left(A_{x}, A_{y}\right) \equiv \frac{m c}{e \hbar}\left(\alpha \sigma_{y}-\beta \sigma_{x},-\alpha \sigma_{x}+\beta \sigma_{y}\right),
$$

we find that the SO interactions and the magnetic gauge $A^{B}$ can be treated in the same manner. The Hamiltonian [Eq. (1)] thus becomes

$$
H=\frac{1}{2 m}\left(\boldsymbol{\Pi}-\frac{e}{c} \mathbf{A}^{\mathrm{SO}}\right)^{2}-V I_{s}=\frac{1}{2 m}\left(\mathbf{p}-\frac{e}{c} \mathbf{A}\right)^{2}-V I_{s},
$$

with the constant potential $V=\left(m / \hbar^{2}\right)\left(\alpha^{2}+\beta^{2}\right)$. The algebra of Pauli matrices $\sigma_{i}^{2}=1$ and $\left\{\sigma_{i}, \sigma_{j}\right\}=2 \delta_{i j}$ with $i, j \in\{x, y, z\}$ is used in arriving at Eq. (3). The unified spin-dependent gauge $\mathbf{A}=\mathbf{A}^{B} I_{s}+\mathbf{A}^{\mathrm{SO}}$ is not a scalar, and its components, in general, do not commute,

$$
\left[A_{x}, A_{y}\right]=2 i\left(\frac{m c}{e \hbar}\right)^{2}\left(\alpha^{2}-\beta^{2}\right) \sigma_{z} .
$$

The presence of the non-Abelian gauge field $\mathbf{A}^{\mathrm{SO}}$, usually referred to as the Yang-Mills field, ${ }^{13}$ makes the usual scalar gauge formalism incapable and complicates the problem. According to Eq. (4), there exists, however, an interesting exception when the RSO and the DSO couplings are of equal strength, i.e., $|\alpha|=|\beta|$. The commutator vanishes also for a one-dimensional system, since only one component of the gauge $\mathbf{A}^{\text {SO }}$ is introduced via Eq. (3). In Sec. III A, we show that both of these cases can be transformed into the case of a free-electron system. In particular, for $|\alpha|=|\beta|$ in the RashbaDresselhaus [001] model, this transformation is a gauge transformation that simplifies the physical picture of the $\mathrm{PSH}^{7,8}$

\section{B. Discrete case: Tight-binding model}

The conventional TB mode $1^{14}$ is based on real crystal structure. Assuming that each electron orbital is localized around its associated nucleus, all spatial interactions involving more than three nuclei centers can thus be neglected. In other words, considering only the nearest-neighbor hopping $t_{\mathbf{m} \sigma \mathbf{m}^{\prime} \sigma^{\prime}}$, from site $\mathbf{m}^{\prime}$ with spin $\sigma^{\prime}$ to site $\mathbf{m}$ with spin $\sigma$, will be sufficient and will be the case exclusively used in the present work. Within this approximation, the Hamiltonian can be represented in the form

$$
\mathcal{H}^{\mathrm{TB}}=\sum_{\mathbf{m} \sigma} u_{\mathbf{m} \sigma} c_{\mathbf{m} \sigma}^{\dagger} c_{\mathbf{m} \sigma}+\sum_{\substack{\left\langle\mathbf{m}, \mathbf{m}^{\prime}\right\rangle \\ \sigma \sigma^{\prime}}} t_{\mathbf{m} \sigma, \mathbf{m}^{\prime} \sigma^{\prime}} c_{\mathbf{m} \sigma^{\prime}}^{\dagger} c_{\mathbf{m}^{\prime} \sigma^{\prime}}
$$

with the on-site energy $u_{\mathbf{m} \sigma}$ tunable by the gate voltage, and the fermion creation (annihilation) operators being denoted by $c_{\mathbf{m} \sigma}\left(c_{\mathbf{m}^{\prime} \sigma^{\prime}}^{\dagger}\right)$, obeying $\left\{c_{\mathbf{m} \sigma}, c_{\mathbf{m}^{\prime} \sigma^{\prime}}^{\dagger}\right\}=1$.

On the other hand, the finite-difference FD method ${ }^{11}$ approximates differentiations by discretizing variables. For example, the differentiation $p_{x} \psi(x)$ in the momentum operator $p_{x}$ is replaced with $-i \hbar\left(\psi_{x+a}-\psi_{x-a}\right) / a \cong-i \hbar d \psi(x) / d x$. Clearly, this approximation is valid only when the wave function $\psi(x)$ varies slowly over one lattice constant $a$ or under the condition $a \ll 1$. Physically, this is the case where only electrons near the band bottom, with small $p=\hbar k$, enter our problem. Despite this restriction, the method is quite general and powerful since it can transform any singleparticle Hamiltonian into a matrix form solvable by numerical methods. Even though the FD method is based on different physical assumptions than the conventional TB model, the single-particle Hamiltonian can always be expressed in the form ${ }^{11}$ of Eq. (5). In particular, it allows us to relate the continuous Hamiltonian [Eq. (1)] to the discrete SOinteracting TB model.

Consider the free-electron system,

$$
H^{\text {free }}=\frac{\Pi^{2}}{2 m} .
$$

The TB Hamiltonian corresponding to Eq. (6) reads ${ }^{11}$

$$
\mathcal{H}^{\mathrm{free}}=\sum_{\mathbf{m} \sigma} c_{\mathbf{m} \sigma}^{\dagger} c_{\mathbf{m} \sigma} u_{\mathbf{m} \sigma}^{\mathrm{free}}+\underset{\substack{\left\langle\mathbf{m}, \mathbf{m}^{\prime}\right\rangle \\ \sigma \sigma^{\prime}}}{\sum} t_{\mathbf{m} \sigma, \mathbf{m}^{\prime} \sigma^{\prime}}^{\text {free }} c_{\mathbf{m} \sigma^{\dagger}}^{\dagger} c_{\mathbf{m}^{\prime} \sigma^{\prime}} .
$$

Each dimension contributes the quantity $2 t_{0} I_{s}$ to the on-site energy, and in a square lattice, we therefore have

$$
u_{\mathbf{m}}^{\text {free }}=4 t_{0} I_{s},
$$

with $u_{\mathbf{m} \sigma}^{\text {free }}=\left\langle\sigma\left|u_{\mathbf{m}}^{\text {free }}\right| \sigma\right\rangle$, the hopping strength defined as $t_{0}$ $=\hbar^{2} / 2 m a^{2}$, and the spin identity matrix denoted by $I_{s}$. The magnetic gauge $\mathbf{A}^{B}$ is introduced as a phase factor in the hopping matrix,

$$
t_{\mathbf{m}, \mathbf{m}^{\prime}}^{\mathrm{free}}=-t_{0} \exp \left[\frac{i e}{c \hbar} \mathbf{A}^{B} \cdot\left(\mathbf{m}-\mathbf{m}^{\prime}\right)\right] I_{s},
$$

with $t_{\mathbf{m} \sigma, \mathbf{m}^{\prime} \sigma^{\prime}}^{\mathrm{free}}=\left\langle\sigma\left|f_{\mathbf{m}, \mathbf{m}^{\prime}}^{\mathrm{free}}\right| \sigma^{\prime}\right\rangle$. We note that the phase acquired by an electron hopping from one site to another over a distance $a$ is proportional to $a \mathbf{A}^{B}$. If we treat the $\mathbf{A}^{\mathrm{SO}}$ as $\mathbf{A}^{B}$ in Eq. (9) and expand the $S U(2)$ phase as

$$
\begin{aligned}
e^{(i e / c \hbar) A_{x}^{\mathrm{SO}} a} e^{(i e / c \hbar) A_{y}^{\mathrm{SO}} a} \approx & e^{i(i e / c \hbar)\left(A_{x}^{\mathrm{SO}}+A_{y}^{\mathrm{SO}}\right) a} \\
& +O\left\{\left(\frac{e a}{c \hbar}\right)^{2}\left[A_{x}^{\mathrm{SO}}, A_{y}^{\mathrm{SO}}\right]\right\},
\end{aligned}
$$

with $\left[A_{x}^{\mathrm{SO}}, A_{y}^{\mathrm{SO}}\right] \equiv A_{x}^{\mathrm{SO}} A_{y}^{\mathrm{SO}}-A_{y}^{\mathrm{SO}} A_{x}^{\mathrm{SO}}$, we find that in the limit, 


$$
\frac{e}{c \hbar} \mathbf{A}^{\mathrm{SO}} \cdot\left(\mathbf{m}-\mathbf{m}^{\prime}\right) \ll 1,
$$

the SO gauge $\mathbf{A}^{\text {SO }}$ plays a similar role as $\mathbf{A}^{B}$ due to their equivalent algebra: $\exp \left[(\right.$ iea $\left./ c \hbar)\left(A_{x}^{B(\mathrm{SO})}\right)\right] \exp [($ iea/ch $)$ $\left.\times\left(A_{x}^{B(\mathrm{SO})}\right)\right]=\exp \left[(\right.$ iea $\left./ c \hbar)\left(A_{x}^{B(\mathrm{SO})}+A_{y}^{B(\mathrm{SO})}\right)\right]$. Accordingly, in the FD approximation with lattice spacing small enough to satisfy Eq. (11), the replacements of

$$
4 t_{0} \rightarrow 4 t_{0}-V
$$

in Eq. (8), and

$$
\mathbf{A}^{B} \rightarrow \mathbf{A}^{B}+\mathbf{A}^{\mathrm{SO}},
$$

in Eq. (9), yield the expression, in the TB form of our SO Hamiltonian [Eq. (3)],

$$
\mathcal{H}=\sum_{\mathbf{m} \sigma} u_{\mathbf{m} \sigma} c_{\mathbf{m} \sigma}^{\dagger} c_{\mathbf{m} \sigma}+\sum_{\substack{\left\langle\mathbf{m}, \mathbf{m}^{\prime}\right\rangle \\ \sigma \sigma^{\prime}}} t_{\mathbf{m} \sigma, \mathbf{m}^{\prime} \sigma^{\prime}} c_{\mathbf{m} \sigma^{\dagger}}^{\dagger} c_{\mathbf{m}^{\prime} \sigma^{\prime}}
$$

but now with $u_{\mathbf{m}}=\left(4 t_{0}-V\right) I_{s}$ and $t_{\mathbf{m}, \mathbf{m}^{\prime}}=$ $-t_{0} \exp \left[(i e / c \hbar) \mathbf{A} \cdot\left(\mathbf{m}-\mathbf{m}^{\prime}\right)\right]$ which takes into account both the background potential $V$ and the SO gauge $\mathbf{A}^{\mathrm{SO}}$.

To make a comparison with the TB Hamiltonian of Ref. 12 where a special case of the square lattice without any applied magnetic field is considered, we let $\mathbf{A}^{B}=0$ and expand the hopping matrix up to the second order of the exponent $(e / c \hbar) \mathbf{A}^{\mathrm{SO}} \cdot\left(\mathbf{m}-\mathbf{m}^{\prime}\right)$,

$$
\begin{aligned}
t_{\mathbf{m}, \mathbf{m}^{\prime}} \cong & -t_{0}\left\{1+\frac{i e}{c \hbar} \mathbf{A}^{\mathrm{SO}} \cdot\left(\mathbf{m}-\mathbf{m}^{\prime}\right)\right. \\
& \left.-\left[\frac{e}{c \hbar} \mathbf{A}^{\mathrm{SO}} \cdot\left(\mathbf{m}-\mathbf{m}^{\prime}\right)\right]^{2}\right\} \\
= & \left\{\begin{array}{l}
-t_{0}\left[1+i \frac{t^{R}}{t_{0}} \sigma_{y}-i \frac{t^{D}}{t_{0}} \sigma_{x}\right]+\frac{V}{2}, \text { for } \mathbf{m}=\mathbf{m}^{\prime}+a \mathbf{e}_{x} \\
-t_{0}\left[1-i \frac{t^{R}}{t_{0}} \sigma_{x}+i \frac{t^{D}}{t_{0}} \sigma_{y}\right]+\frac{V}{2}, \text { for } \mathbf{m}=\mathbf{m}^{\prime}+a \mathbf{e}_{y}
\end{array}\right.
\end{aligned}
$$

where the RSO and DSO hoppings are, respectively, defined by $t^{R}=\alpha / 2 a$ and $t^{D}=\beta / 2 a$. Moreover, in the limit of Eq. (11), we can approximate the operation $-V \psi_{\mathbf{m}}$ as $-V$ $\left(\psi_{\mathbf{m}+a \mathbf{e}_{i}}+\psi_{\mathbf{m}-a \mathbf{e}_{i}}\right) / 2$ with $i \in\{x, y\}$ due to the slow variation of the wave function $\psi_{\mathbf{m}} \cong\left(\psi_{\mathbf{m}+a \mathbf{e}_{i}}+\psi_{\mathbf{m}-a \mathbf{e}_{i}}\right) / 2$. This approximation converts the on-site background potential $-V$ into the hopping $-V / 2$ and thus cancels out the last term $V / 2$ in Eq. (15). Finally, in Eq. (14), we have $u_{\mathbf{m}}=4 t_{0} I_{s}, t_{\mathbf{m}, \mathbf{m}^{\prime}}=-t_{0}[1$ $\left.+i\left(t^{R} / t_{0}\right) \sigma_{y}-i\left(t^{D} / t_{0}\right) \sigma_{x}\right]$ for $\mathbf{m}=\mathbf{m}^{\prime}+a \mathbf{e}_{x}$, and $t_{\mathbf{m}, \mathbf{m}^{\prime}}=-t_{0}[1$ $\left.-i\left(t^{R} / t_{0}\right) \sigma_{x}+i\left(t^{D} / t_{0}\right) \sigma_{y}\right]$ for $\mathbf{m}=\mathbf{m}^{\prime}+a \mathbf{e}_{y}$. By further letting $t^{D}=0$, Eq. (14) reduces to the form adopted in Ref. 12. This suggests that, in the FD approximation and the limit of Eq. (11), it is reasonable to treat the SO gauge as a magnetic one.

For the 2DEG made of InGaAs/InAlAs heterostructure grown along [001] direction, the typical parameters ${ }^{1}$ are the effective electron mass $m \approx 0.05 m_{e}$ ( $m_{e}$ is the electron mass), the SO coupling strength $\approx 0.3 \mathrm{eV} \AA$, and the lattice constant $a \approx 3 \mathrm{~nm}$. These quantities yield the desired small value $(e / c \hbar) \mathbf{A}^{\mathrm{SO}} \cdot\left(\mathbf{m}-\mathbf{m}^{\prime}\right) \approx 1.5 \times 10^{-3}$ so that Eq. (11) indeed holds. Moreover, if Eq. (11) is satisfied, then the $S U(2)$ gauge can be approximated as the $U(1)$ gauge. Accordingly, two general properties of the TB Hamiltonian [Eq. (14)] can be noted. First, the sites $\mathbf{m}$ are not necessarily arranged on a square lattice, i.e., one can apply Eq. (14) to the hopping along an arbitrary direction. Second, for spatially dependent (nonuniform) SO interactions ${ }^{15} \alpha=\alpha(\mathbf{r})$ and $\beta=\beta(\mathbf{r})$, the hopping exponent $\mathbf{A}^{\mathrm{SO}} \cdot\left(\mathbf{m}-\mathbf{m}^{\prime}\right)$ can simply be replaced with $\int_{\mathbf{m}^{\prime}}^{\mathbf{m}} \mathbf{A}^{\mathrm{SO}}\left(\mathbf{r}^{\prime}\right) \cdot d \mathbf{r}^{\prime}$ and the formalism remains the same.

\section{APPLICATIONS}

In Sec. III A, we present the application of the continuous case based on Eq. (3), and in Sec. III B, we present the application of the discrete case based on Eq. (14). The first application is a study of the PSH from the gauge transformation, and the second extends the previous work of Ref. 10 on the quantum square ring.

\section{A. Continuous case: Gauge transformation and persistent spin helix}

Consider the local transformation operator $U(\mathbf{r})$ $=\exp \left[(i e / \hbar c) \int_{c} \mathbf{A}^{\mathrm{SO}}\left(\mathbf{r}^{\prime}\right) \cdot d \mathbf{r}^{\prime}\right]$. In general, $U(\mathbf{r})$ depends on the integration path $c$. Nevertheless, in the case of uniform Rashba and Dresselhaus interactions, $\mathbf{A}^{\mathrm{SO}}$ is independent of the position $\mathbf{r}=(x, y)$, leading to $\nabla \times \mathbf{A}^{\mathrm{SO}}=0$ so that $\int_{c} \mathbf{A}^{\mathrm{SO}} d \mathbf{r}^{\prime}=\mathbf{A}^{\mathrm{SO}} \cdot \mathbf{r}$ becomes path independent. This yields the expression

$$
U(\mathbf{r})=\exp \left[\frac{i e}{\hbar c}\left(\mathbf{A}^{\mathrm{SO}} \cdot \mathbf{r}\right)\right]
$$

with the unitary property $U(\mathbf{r}) U^{\dagger}(\mathbf{r})=I_{s}$ ensured by the Hermitian relation $\mathbf{A}^{\mathrm{SO} \dagger}=\mathbf{A}^{\mathrm{SO}}$, which follows from the definition [Eq. (2)]. We notice that Eq. (3) differs from the free-electron gas (with a background potential $V$ ),

$$
h=\frac{\boldsymbol{\Pi}^{2}}{2 m}-V,
$$

only by a gauge $(e / c) \mathbf{A}^{\mathrm{SO}}$. This suggests to the transformation,

$$
\begin{aligned}
U(\mathbf{r}) \boldsymbol{\Pi} U^{\dagger}(\mathbf{r})= & \boldsymbol{\Pi}+\frac{i e}{\hbar c}\left[\mathbf{A}^{\mathrm{SO}} \cdot \mathbf{r}, \boldsymbol{\Pi}\right] \\
& +\frac{1}{2}\left(\frac{i e}{\hbar c}\right)^{2}\left\{\mathbf{A}^{\mathrm{SO}} \cdot \mathbf{r},\left[\mathbf{A}^{\mathrm{SO}} \cdot \mathbf{r}, \boldsymbol{\Pi}\right]\right\}+\cdots,
\end{aligned}
$$

with $\left[\mathbf{A}^{\mathrm{SO}} \cdot \mathbf{r}, \mathbf{\Pi}\right]=i \hbar \mathbf{A}^{\mathrm{SO}}$. However, due to the nonvanishing commutator $\left[A_{x}^{\mathrm{SO}}, A_{y}^{\mathrm{SO}}\right] \neq 0$, the higher order terms in Eq. (18), in general, do not vanish, leading to $U(\mathbf{r}) h U^{\dagger}(\mathbf{r}) \neq H$. 
In the exceptional equal-strength case $|\alpha|=|\beta|$, the operators $A_{x}^{\mathrm{SO}}$ and $A_{y}^{\mathrm{SO}}$ satisfy the scalar algebra $A_{x}^{\mathrm{SO}} A_{y}^{\mathrm{SO}}$ $=A_{y}^{\mathrm{SO}} A_{x}^{\zeta \mathrm{O}}$, and hence we obtain $U(\mathbf{r}) \Pi U^{\dagger}(\mathbf{r})=\Pi-e / c \mathbf{A}^{\mathrm{SO}}$ and the gauge transformation,

$$
U^{\dagger}(\mathbf{r}) H U(\mathbf{r})=h .
$$

As a result, the free-electron gas $h$ in Eq. (17) and the SOinteracting electron gas $H$ in Eq. (3) share the same eigenenergies $E_{\mathbf{k}}$. Their corresponding eigenfunctions, denoted by $\psi_{E_{\mathbf{k}}}(\mathbf{r}) \chi_{s}^{\text {free }}$ and $\Psi_{E_{\mathbf{k}}}(\mathbf{r}) \chi_{s}^{\mathrm{SO}}$, respectively, differ from each other only by a phase factor defined by the $2 \times 2$ matrix $U(\mathbf{r})$, namely, $\Psi_{E_{\mathbf{k}}}(\mathbf{r}) \chi_{s}^{\mathrm{SO}}=U(\mathbf{r}) \psi_{E_{\mathbf{k}}}(\mathbf{r}) \chi_{s}^{\text {free }}$. Moreover, any wave function is constructed by a superposition of the eigenfunctions, so that for any given wave function $\psi(\mathbf{r}) \chi_{s}^{\text {free }}$ in $h$, the corresponding wave function in $H$ is $U(\mathbf{r}) \psi(\mathbf{r}) \chi_{s}^{\text {free }}$. Below, we show that the physical description of the PSH in the SO-interacting system can be easily understood by this correspondence.

Consider first an injected electron in the system $h$, described by $\psi_{\text {inj }}(\mathbf{r}) \chi_{\text {inj }}=\left[\Sigma_{\mathbf{k}} C_{\mathbf{k}} \psi_{E_{\mathbf{k}}}(\mathbf{r})\right] \chi_{\text {inj }}$, with the initial spin state $\chi_{\text {inj }}$ and the weight factor $C_{\mathbf{k}}$. Clearly, without any spindependent mechanisms, this electron retains its spin state $\chi_{\text {inj }}$ as it traverses the sample. We introduce now the factor $\mathbf{A}^{\mathrm{SO}}$. This corresponds to turning on $U(\mathbf{r})$ so that the electron wave function, in the SO-interacting system $H$, undergoes the gauge transformation of $U(\mathbf{r})$,

$$
U(\mathbf{r}) \psi_{\text {inj }}(\mathbf{r}) \chi_{\text {inj }}=\sum_{\mathbf{k}} C_{\mathbf{k}} \psi_{E_{\mathbf{k}}}(\mathbf{r}) U(\mathbf{r}) \chi_{\text {inj }}
$$

Accordingly, the spin polarization of the electron varies spatially according to $U(\mathbf{r}) \chi_{\text {inj. }}$.

Using Eqs. (16) and (2), with $\alpha=\beta$, we find that

$$
\begin{aligned}
\left.U(\mathbf{r})\right|_{\alpha=\beta} & =\exp \left[-\alpha \frac{i 2 m}{\hbar^{2}} \sigma_{(1,-1)} r_{(1,1)}\right] \\
& =\exp \left[-i \frac{\hbar \sigma_{(1,-1)} / 2}{\hbar} \theta_{\mathrm{PSH}}^{+}\right],
\end{aligned}
$$

where $\sigma_{(1,-1)} \equiv\left(\sigma_{x}, \sigma_{y}\right) \cdot(1,-1) / \sqrt{2}$ is actually the spin rotation operator, with rotation axis along $(1,-1)$ and the precession angle $\theta_{\mathrm{PSH}}^{+} \equiv\left(4 \alpha m / \hbar^{2}\right) r_{(1,1)}$ depending on the distance $r_{(1,1)} \equiv \mathbf{r} \cdot(1,1) / \sqrt{2}$ along $(1,1)$ [cf. Fig. 2(b) in Ref. 8]. Similarly, for $\alpha=-\beta$, we have

$$
\left.U(\mathbf{r})\right|_{\alpha=-\beta}=\exp \left[-i \frac{\hbar \sigma_{(1,1)} / 2}{\hbar} \theta_{\mathrm{PSH}}^{-}\right],
$$

corresponding to a rotation axis along $(1,1)$ and a precession angle $\theta_{\mathrm{PSH}}^{-}=\left(4 \alpha m / \hbar^{2}\right) r_{(-1,1)}$ with $r_{(-1,1)} \equiv \mathbf{r} \cdot(-1,1) / \sqrt{2}$.

The equal-strength case, $\alpha=\beta$ or $\alpha=-\beta$, shown above is precisely the PSH with precession length,

$$
L_{P}=\frac{\hbar^{2} \pi}{2 m \alpha},
$$

for spin to rotate $\theta_{\mathrm{PSH}}^{+}$or $\theta_{\mathrm{PSH}}^{-}=2 \pi$. Obviously, the PSH is robust against any spin-independent mechanisms for which $U^{\dagger}(\mathbf{r}) V_{p}(\mathbf{r}) U(\mathbf{r})=V_{p}(\mathbf{r})$ holds for any potential of the form $V_{p}(\mathbf{r}) \propto I_{s}$. This property is satisfied also by the finite-size (a)

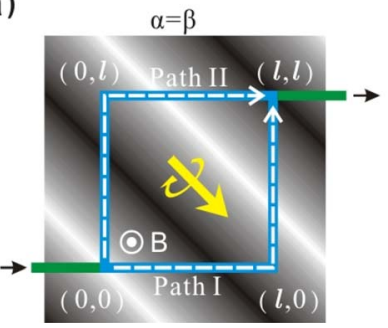

(b)

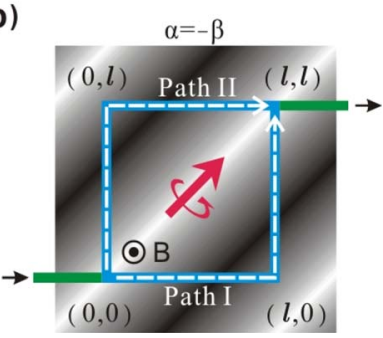

FIG. 1. (Color online) A square ring with corners at $(0,0),(l, 0)$, $(l, l)$, and $(l, l)$, in contact with two leads. A magnetic field $\mathbf{B}=B \mathbf{e}_{z}$ is locally applied at the center of the ring. Electrons are injected from the left lead and then transmitted to the right lead via path I and path II. The background shading represents the helix precession angle. From white to black, the electron precesses by the angle $\pi$. The rotation axes are specified by the arrows at the centers of the rings for (a) $\alpha=\beta$ and (b) $\alpha=-\beta$.

confinement due to spin-independent boundaries. In particular, the presence of $\mathbf{A}^{B}$, which determines the actual form of $\psi_{E_{\mathbf{k}}}(\mathbf{r})$, clearly does not affect the PSH. This is reasonable since $\mathbf{A}^{B}$ contributes only a spin-independent phase to the electron wave function due to the absence of the Zeeman term and therefore does not vary the spin polarization. Inclusion of the Zeeman term requires further generalization, which is beyond the scope of the present discussion.

A similar calculation can also apply to the DSO [110] linear model,

$$
H_{[110]}=\frac{p_{x}^{2}+p_{y}^{2}}{2 m}-\frac{2 \beta}{\hbar} p_{x} \sigma_{z}
$$

with the gauge $\quad \mathbf{A}_{[110]}^{\mathrm{SO}}=\left(A_{x[110]}, A_{y[110]}, 0\right)$ $\equiv\left(2 \beta \sigma_{z}, 0,0\right) m c / e \hbar$. Due to $\left[A_{x[110]}, A_{y[110]}\right]=0$, we have here the PSH described by

$$
U(\mathbf{r})_{[110]}=\exp \left(-i \frac{\hbar \sigma_{z} / 2}{\hbar} \theta_{\mathrm{PSH}}\right),
$$

with $\theta_{\mathrm{PSH}}=-4 m \beta x / \hbar^{2}$ [cf. Fig. 2(d) in Ref. 8].

We further point out that a one-dimensional SOinteracting system (with any values of $\alpha$ and $\beta$ ) is also amenable to this transformation. For example, consider a onedimensional (along $\mathbf{e}_{n}$ direction) SO-interacting conductor. Since the degrees of freedom in the orthogonal directions are frozen, only $\Pi_{n} \equiv \boldsymbol{\Pi} \cdot \mathbf{e}_{n}$ and $A_{n}^{\mathrm{SO}} \equiv \mathbf{A}^{\mathrm{SO}} \cdot \mathbf{e}_{n}$ are relevant to our problem and appear in Eq. (3). The system can now be described by $H^{1 \mathrm{D}}=\left[\Pi_{n}-(e / c) A_{n}^{\mathrm{SO}}\right]^{2} /(2 m)-V^{1 \mathrm{D}}$, with a constant potential $V^{1 \mathrm{D}}$. Without encountering the noncommutability [Eq. (4)], we arrive again, by a procedure similar to those above, at the transformation $U^{1 \mathrm{D} \dagger}\left(r_{n}\right) H^{1 \mathrm{D}} U^{1 \mathrm{D}}\left(r_{n}\right)$ $=h^{1 \mathrm{D}}=\Pi_{n}^{2} / 2 m-V^{1 \mathrm{D}}$, with $U^{1 \mathrm{D}}\left(r_{n}\right)=\exp \left[(i e / \hbar c) A_{n}^{\mathrm{SO}} \cdot r_{n}\right]$ and $r_{n} \equiv \mathbf{r} \cdot \mathbf{e}_{n}$.

\section{B. Discrete case: Four states in the quantum square ring}

Using the TB Hamiltonian of Eq. (14), we investigate in this section a square ring interferometer ${ }^{10}$ (see Fig. 1) under the influence of a magnetic field and of the DSO and RSO 


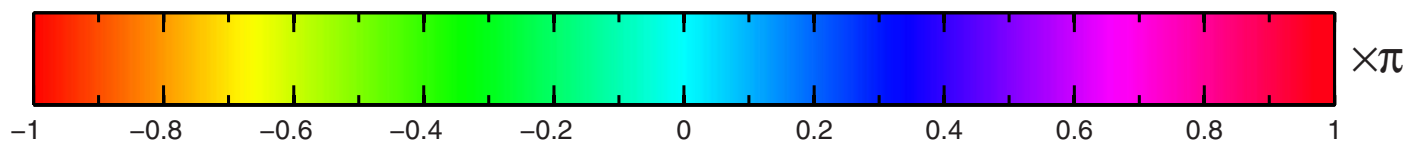

(a)

(b)

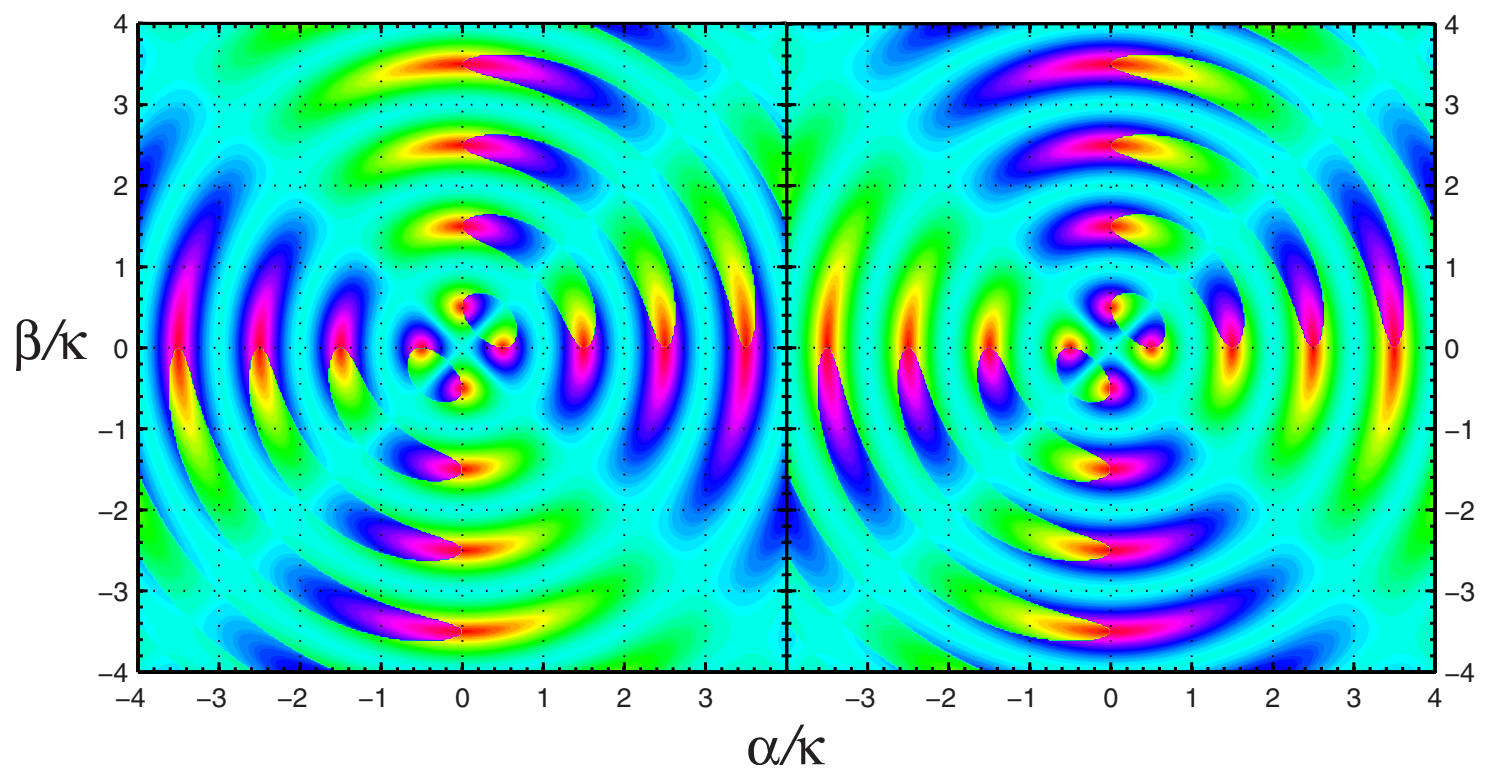

FIG. 2. (Color online) Spin-orbit phases in (a) $\phi_{\uparrow}^{\mathrm{SO}}$ and (b) $\phi_{\tilde{\tau}}^{\mathrm{SO}}$ as functions of $\alpha / \kappa$ and $\beta / \kappa$ with $\kappa \equiv \pi \hbar^{2} / m l$ for the square ring patterned in the Rashba-Dresselhaus [001] 2DEG.

couplings. The transmission coefficients $T_{\sigma^{\prime} \sigma}$, with incoming (outgoing) spin denoted by $\sigma\left(\sigma^{\prime}\right)$ depend on the interplay among the phases due to the RSO and DSO interactions and the external field. Depending on the values of the magnetic field $B$, the Rashba coefficient $\alpha$, and Dresselhaus coefficient $\beta$, there appear "perfect" four states in this setup: insulating, spin-filtering, ${ }^{10,16,17}$ spin-flipping, and spin-keeping states. When electrons are injected into the system, the spininsulating ring blocks both up and down spin channels, the spin-filtering ring blocks only one of the spin channels, the spin-flipping ring flips the spins of the injected electrons, and the spin-keeping ring keeps the injected spin configuration unaltered. It is worth mentioning that these four states are perfect meaning that they are valid for any range of the injected energy $E$.

Consider a square ring (see Fig. 1) described by $\mathcal{H}$ in Eq. (14), with width $l$ and corners at $\mathbf{m}=(0,0),(l, 0),(l, l)$, and $(0, l)$. In the center hollow region, we apply the magnetic field $\mathbf{B}=B \mathbf{e}_{z}$. The field penetrates the ring, but it is not applied to the ring itself. Thus, the Zeeman splitting due to the magnetic field can be disregarded. Two ideal leads contact the ring at the positions $(0,0)$ and $(l, l)$. For brevity, and without loss of generality, we choose the on-site energy in both leads and the ring to be zero. The self-energy ${ }^{11} \Sigma(E)=(E$ $\left.-i \sqrt{4 t_{0}^{2}-E^{2}}\right)\left(c_{(0,0)}^{\dagger} c_{(0,0)}+c_{(l, l)}^{\dagger} c_{(l, l)}\right) / 2$ is generated due to the presence of the leads. The transmission coefficients are computed by

$$
T_{\sigma^{\prime} \sigma}=\left\langle(l, l) ; \sigma^{\prime}\left|\frac{\sqrt{4 t_{0}^{2}-E^{2}}}{E-\mathcal{H}-\Sigma(E)}\right|(0,0) ; \sigma\right\rangle,
$$

with the incoming (from left lead) spin $\sigma$ and outgoing (to the right lead) spin $\sigma^{\prime}$ states denoted as $|(0,0) ; \sigma\rangle$ and $\left|(l, l) ; \sigma^{\prime}\right\rangle$, respectively. The interference between the bottom-right path $\mathrm{I} \equiv(0,0) \rightarrow(l, 0) \rightarrow(l, l)$ and the left-top path $\mathrm{II} \equiv(0,0) \rightarrow(0, l) \rightarrow(l, l)$ is determined by the phase circling the ring of the form $U_{\text {phase }}=U_{\text {II }}^{\dagger} U_{\mathrm{I}}$ $=\left[U_{(l, l) \leftarrow(0, l)} U_{(0, l) \leftarrow(0,0)}\right]^{\dagger} U_{(l, l) \leftarrow(l, 0)} U_{(l, 0) \leftarrow(0,0)}$

$=U_{(0,0) \leftarrow(0, l)} U_{(0, l) \leftarrow(l, l)} U_{(l, l) \leftarrow(l, 0)} U_{(l, 0) \leftarrow(0,0)} \quad$ with $\quad U_{\mathbf{m}^{\prime} \leftarrow \mathbf{m}}$ $\equiv \exp \left[(i e / c \hbar) \mathbf{A} \cdot\left(\mathbf{m}-\mathbf{m}^{\prime}\right)\right]$, where $\mathbf{A}=\mathbf{A}^{B} I_{s}+\mathbf{A}^{\mathrm{SO}}$ accounts for the magnetic gauge $\mathbf{A}^{B}=(-B y, B x, 0) / 2$ and the $\mathrm{SO}$ gauge of Eq. (2). Explicitly, in the square ring, we obtain the $2 \times 2$ matrix,

$$
\begin{aligned}
U_{\text {phase }}= & e^{i \phi^{B}} e^{i\left(m l / \hbar^{2}\right)\left(\alpha \sigma_{x}-\beta \sigma_{y}\right)} e^{-i\left(m l / \hbar^{2}\right)\left(\alpha \sigma_{y}-\beta \sigma_{x}\right)} \\
& \times e^{-i\left(m l / \hbar^{2}\right)\left(\alpha \sigma_{x}-\beta \sigma_{y}\right)} e^{i\left(m l / \hbar^{2}\right)\left(\alpha \sigma_{y}-\beta \sigma_{x}\right)},
\end{aligned}
$$

corresponding to the magnetic flux $\phi^{B}=(e / c \hbar) B l^{2}$. Denote the general spin-up state with the polar angle $\theta \in[0, \pi]$ and azimuthal angle $\phi \in[0,2 \pi]$ as $\uparrow_{[\theta, \phi]}$, i.e., 


$$
\uparrow_{[\theta, \phi]}=\left(\begin{array}{c}
e^{-i \phi / 2} \cos \frac{\theta}{2} \\
e^{i \phi / 2} \sin \frac{\theta}{2}
\end{array}\right) .
$$

Strictly speaking, in the arbitrary spin-up $\uparrow_{[\theta, \phi]}$ and spindown $\downarrow_{[\theta, \phi]}$ axes, $U_{\text {phase }}$ does not give us the meaning of "phase," since it is, in general, a nondiagonal matrix, while in the diagonal axes with

$$
D^{\dagger} U_{\text {phase }} D=\Lambda,
$$

defining the tilted up $|\widetilde{\uparrow}\rangle$ (down $|\widetilde{\downarrow}\rangle$ ) spin as $|\widetilde{\uparrow}\rangle=D\left|\uparrow_{[0,0]}\right\rangle$ $\left(|\widetilde{\downarrow}\rangle=D\left|\downarrow_{[0,0]}\right\rangle\right)$, the circled phase $\boldsymbol{\phi}_{\uparrow(\tilde{\downarrow})}$ acquired by $|\widetilde{\uparrow}\rangle(|\widetilde{\downarrow}\rangle)$ electron can be read off via the eigenvalues of $U_{\text {phase}}$,

$$
\Lambda=\left(\begin{array}{cc}
e^{i \phi \tilde{\uparrow}} & 0 \\
0 & e^{i \phi_{\downarrow}}
\end{array}\right),
$$

with $\phi_{\uparrow(\tilde{\complement})} \equiv \phi^{B}+\phi_{\tilde{\uparrow}(\tilde{\downarrow})}^{\mathrm{SO}}$ contributed by both magnetic $\phi^{B}$ and SO $\phi_{\tau(\tilde{\downarrow})}^{\text {SO }}$ phases. Consider now a special case with vanishing $\beta, \phi^{B}=\pi / 2$, and $\phi_{\tilde{\uparrow}(\tilde{\downarrow})}^{\mathrm{SO}}=+(-) \pi / 2$. This corresponds to the Rashba strength, ${ }^{10}$

$$
\alpha^{*}=\frac{\hbar^{2}}{m l} \sin ^{-1}\left(2^{-1 / 4}\right)
$$

There is, therefore, destructive interference $e^{i \phi \tilde{\uparrow}}=e^{i \pi}=-1$ for $|\widetilde{\uparrow}\rangle$ electrons and constructive interference $e^{i \phi \tilde{\downarrow}}=e^{i 0}=1$ for $|\tilde{\downarrow}\rangle$ electrons. The spin-filtering ring (filtering out $|\widetilde{\uparrow}\rangle$ ) is thus achieved in this case.

Note that there is no absolute "up" and "down" directions for tilted spins. For the two eigenvalues, $\lambda_{1}$ and $\lambda_{2}$, of Eq. (27), one can select $\lambda_{1}$ as the first eigenvalue $\Lambda_{11}$ $=\exp \left(i \phi_{\uparrow}\right)=\lambda_{1}$ and $\lambda_{2}$ as the second $\Lambda_{22}=\exp \left(i \phi_{\uparrow} \tilde{)}=\lambda_{2}\right.$ to construct the rotation matrix $D$, while it is also allowed to select $\lambda_{2}$ as the first eigenvalue and $\lambda_{1}$ as the second, i.e., $\exp \left(i \phi_{\uparrow}\right)=\lambda_{2}$ and $\exp \left(i \phi_{\downarrow}\right)=\lambda_{1}$ which yields another $D$. This selectivity does not cause any confusion, since the tilted-up spin $|\widetilde{\uparrow}\rangle$ and tilted-down spin $|\widetilde{\downarrow}\rangle$ are also switched if one swaps the eigenvalues. As a result, as long as the constructed $D$ is consistent with the definition [Eq. (29)], the phases [or $\exp \left(i \phi_{\uparrow}\right)$ and $\left.\exp \left(i \phi_{\urcorner}\right)\right]$are uniquely determined for $|\widetilde{\uparrow}\rangle$ and $|\widetilde{\downarrow}\rangle$. We now numerically analyze the SO phase $\phi_{\widetilde{\uparrow}(\widetilde{\downarrow})}^{\mathrm{SO}}$ and the transmission coefficients $T_{\sigma^{\prime} \sigma}$ in Eq. (26).

In Figs. 2(a) and 2(b), we plot the tilted-spin-up phase $\phi_{\uparrow}^{\mathrm{SO}}$ and tilted-spin-down phase $\phi_{\tau}^{\mathrm{SO}}$ as functions of the Rashba $\alpha / \kappa$ and Dresselhaus $\beta / \kappa$ interaction strengths with normalization factor $\kappa \equiv \pi \hbar^{2} / m l$ depending on the length $l$ of the square ring. Since $U_{\text {phase }}\left(\phi_{B} \rightarrow 0\right)$ in Eq. (27) is a rotation in the $S U(2)$ group, we have $\operatorname{det}\left[U_{\text {phase }}\left(\phi_{B} \rightarrow 0\right)\right]$ $=\operatorname{det} \Lambda=1$, yielding $\phi_{\uparrow}^{\mathrm{sO}}=-\phi_{\tilde{\downarrow}}^{\mathrm{SO}}$ which is clearly seen in Fig. 2. Furthermore, under the replacement $(\alpha, \beta) \rightarrow(\beta, \alpha)$, i.e., swapping $\alpha$ and $\beta$, with selecting the same rotation matrix $D$, the SO phases are antisymmetric (different by a sign). Nodes are also found in the phases (Fig. 2). First, along $|\alpha|$

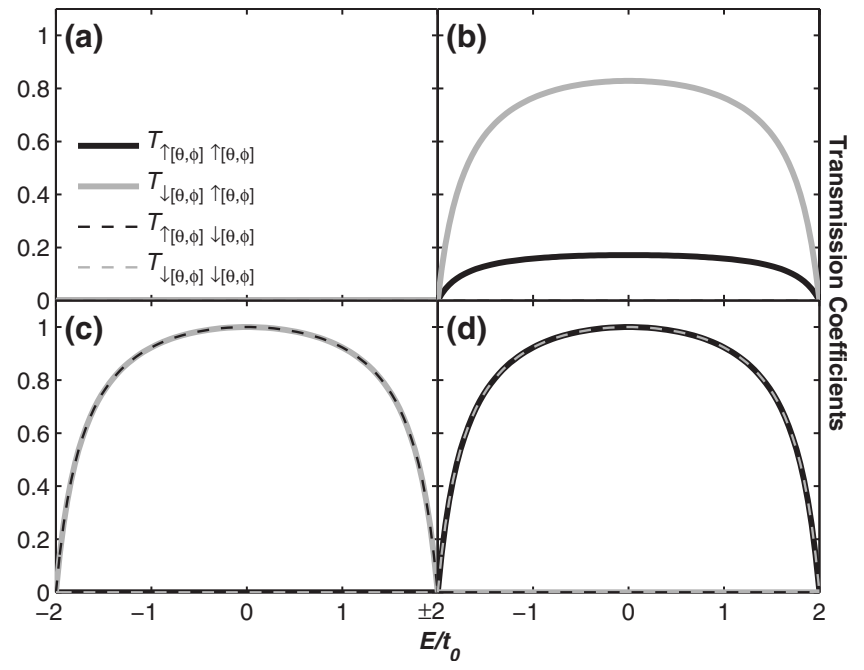

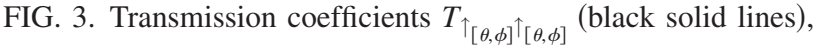

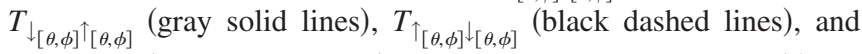
$T_{\downarrow_{[\theta, \phi]} \downarrow_{[\theta, \phi]}}$ (gray dashed lines) as functions of energy $E$. In (a) the insulating state and (d) the spin-keeping state, the up spin $\uparrow_{[\theta, \phi]}$ is defined by Eq. (28) for any $\theta \in[0, \pi]$ and $\phi \in[0,2 \pi]$. In (b) the spin-filtering state, up spin is defined in the tilted axes mentioned in Sec. III B, i.e., $\uparrow_{[\theta, \phi]} \equiv \widetilde{\uparrow}$, whereas in (c) the spin-flipping state, it is defined in any directions lying in the $x=y$ plane, i.e., $\uparrow[\theta, \phi]$ $\equiv \uparrow[\theta, \pi / 4]$.

$=|\beta|, U_{\text {phase }}\left(\phi_{B} \rightarrow 0,|\alpha| \rightarrow|\beta|\right)=I_{s}$ gives zero $\phi_{\widetilde{\uparrow}(\widetilde{)})}^{\text {SO }}$. Second, along the circles $R_{s}=\sqrt{\left(\alpha^{2}+\beta^{2}\right) / \kappa^{2}}=s$, with $s=0,1,2, \cdots$, $\phi_{\uparrow(\tilde{\downarrow})}^{\mathrm{SO}}$ vanishes. These nodes can actually be obtained by a direct analytical computation on the eigenvalues of Eq. (27) with imposing the condition of positive real eigenvalues.

Figure 3 plots the transmission coefficients $T_{\sigma^{\prime} \sigma}$, with $\sigma$, $\sigma^{\prime}=\{\widetilde{\uparrow}, \widetilde{\downarrow}\}$, as functions of the injection energy $E$. Obviously, for any $|\alpha|=|\beta|$ with $\phi^{B}=(2 n+1) \pi$ and $n \in$ integer, the ring is completely destructive for electron spins of any directions due to $\phi_{\uparrow[\theta, \phi]}=\phi_{\downarrow[\theta, \phi]}=(2 n+1) \pi$. In Fig. 3(a), we thus have the insulating state. For the spin-filtering state in Fig. 3(b), we take the values $(\alpha, \beta) / \alpha^{*}=(0,1)$ and $\phi^{B}=(4 n+1) \pi / 2$ which contribute to the $\widetilde{\uparrow}$ and $\tilde{\downarrow}$ spins, respectively, with the constructive $\phi_{\uparrow}=2 n \pi$ and destructive $\phi_{\uparrow}=(2 n+1) \pi$ phases. As a response to the antisymmetric property of the $\phi_{\uparrow(\tilde{\downarrow})}$ mentioned above, the indices $\widetilde{\uparrow}$ and $\tilde{\downarrow}$ in the transmission coefficients are swapped if we interchange $\alpha$ and $\beta$, i.e., choose the values $(\alpha, \beta) / \alpha^{*}=(1,0)$ as used in Ref. 10 .

Here, the destructive interference means that the outgoing channel is blocked. So far, we have not utilized any relation between the incoming and outgoing spin states. These two states are correlated with each other by the spin precession, for example, the PSH, which yields the spin-flipping ring in Fig. 3(c) and spin-keeping ring in Fig. 3(d). We now focus on the case of $|\alpha|=|\beta|$. Without loss of generality, assume $\alpha>0$ for simplicity. Due to $U_{\text {phase }}(|\alpha| \rightarrow|\beta|) \propto I_{s}$, the phases are now merely determined by the magnetic gauge and become spin independent, i.e., $\phi^{B}=\phi_{\uparrow_{[\theta, \phi]}}=\phi_{\downarrow_{[\theta, \phi]}}$. As a result, the interference and the precession are decoupled in the PSH 
and are governed separately by the magnetic and the SO gauges.

In one of the equal-strength case, namely, $\alpha=\beta$, we have, according to Eq. (21), the PSH pattern shown in Fig. 1(a). The condition $\sqrt{2} l=L_{P}(2 s+1) / 2$, with $L_{P}$ defined in Eq. (23), assumes the form

$$
\alpha=\beta=\frac{(2 s+1) \pi \hbar^{2}}{4 \sqrt{2} l m}
$$

and rotates any injected spin $\uparrow_{[\theta, \pi / 4]}$ (or $\downarrow_{[\theta, \pi / 4]}$ ) along the $x$ $=y$ plane by the angle $\theta_{\mathrm{PSH}}^{+}=\pi(2 s+1)$. For example, the sketch in Fig. 1(a) is the case with $\theta_{\mathrm{PSH}}^{+}=3 \pi$. As a result, after passing through the ring, $\uparrow_{[\theta, \pi / 4]}$ is flipped to $\downarrow_{[\theta, \pi / 4]}$ (or $\downarrow_{[\theta, \pi / 4]}$ to $\left.\uparrow_{[\theta, \pi / 4]}\right)$. This represents the spin-flipping ring. Indeed, under the condition (32), and with $B=0$, our numerical result in Fig. 3(c) suggests the spin-flipping state by showing vanishing $T_{\left[[\theta, \pi / 4]^{\uparrow}[\theta, \pi / 4]\right.}$ and $T_{\downarrow_{[\theta, \pi / 4]} \downarrow_{[\theta, \pi / 4]}}$ and nonvanishing

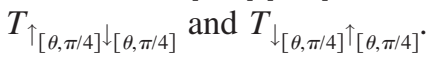

In the other equal-strength case, $\alpha=-\beta$, we have, according to Eq. (22), the PSH pattern shown in Fig. 1(b). Therefore, as long as the ring retains its square shape, injected spin of arbitrary direction remains in its original configuration due to the zero precession angle $\theta_{\mathrm{PSH}}=0$. One thus arrives at the spin-keeping ring in accord with Fig. 3(d) which predicts

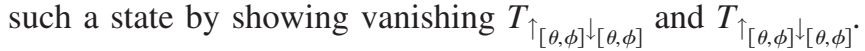
Note that although Figs. 3(c) and 3(d) are plotted for the special case $B=0$, the features of spin flipping and spin keep- ing are robust against magnetic field since they result from precession rather than phase change.

\section{SUMMARY}

In the limit of Eq. (11), the approximation [Eq. (10)] suggests that the $S U(2)$ or $\mathrm{SO}$ phases can be treated as $U(1)$ phases. We justified the SO-interacting TB model, established from this analogy, by comparison with one previously published result given in Ref. 12. The PSH, initially obtained from a global $S U(2)$ transformation, ${ }^{7}$ is obtained here by means of a local gauge transformation. As an application of the geometry of the PSH pattern, we considered a square ring in contact with two ideal leads. This setup is found to be a versatile spintronics device performing four types of functions in which spins are insulated, filtered, flipped, or kept (in polarization). The former two are due to phase change, while the latter two are due to spin precession and newly proposed here. The SO phases in the presence of both RSO and DSO couplings are also analyzed. In particular, the SO phases are antisymmetric under the exchange of these two SO couplings. Our numerical results on transmission coefficients conform with theoretical predictions on the PSH and thus suggest a four state device.

\section{ACKNOWLEDGMENTS}

One of the authors (S.-H.C.) thanks Ming-Hao Liu for valuable discussions and suggestions. This work is supported by the Republic of China National Science Council under Grant No. 95-2112-M-002-044-MY3. *d92222006@ntu.edu.tw

${ }^{1}$ J. Nitta, T. Akazaki, H. Takayanagi, and T. Enoki, Phys. Rev. Lett. 78, 1335 (1997).

${ }^{2}$ B. Das, D. C. Miller, S. Datta, R. Reifenberger, W. P. Hong, P. K. Bhattacharya, J. Singh, and M. Jaffe, Phys. Rev. B 39, R1411 (1989).

${ }^{3}$ E. I. Rashba, Sov. Phys. Solid State 2, 1109 (1960).

${ }^{4}$ G. Dresselhaus, Phys. Rev. 100, 580 (1955).

${ }^{5}$ G. Lommer, F. Malcher, and U. Rössler, Phys. Rev. B 32, 6965 (1985); Yu. A. Bychkov and E. I. Rashba, in Proceedings of the 17th International Conference on Physics of Semiconductors, San Francisco, 1984 (Springer, New York, 1985), p. 321; M. I. D'yakonov and V. Y. Kachorovskii, Sov. Phys. Semicond. 20, 110 (1986).

${ }^{6}$ I. Žutić, J. Fabian, and S. Das Sarma, Rev. Mod. Phys. 76, 323 (2004).

${ }^{7}$ B. A. Bernevig, J. Orenstein, and S. C. Zhang, Phys. Rev. Lett. 97, 236601 (2006).

${ }^{8}$ Ming-Hao Liu, Kuo-Wei Chen, Son-Hsien Chen, and Ching-Ray
Chang, Phys. Rev. B 74, 235322 (2006).

${ }^{9}$ J. Schliemann, J. C. Egues, and D. Loss, Phys. Rev. Lett. 90, 146801 (2003).

${ }^{10}$ N. Hatano, R. Shirasaki, and H. Nakamura, Phys. Rev. A 75, 032107 (2007).

${ }^{11}$ S. Datta, Electronic Transport in Mesoscopic Systems (Cambridge University Press, Cambridge, 1995), Sec. 3.5.

${ }^{12}$ B. K. Nikolic, L. P. Zârbo, and S. D. Souma, Phys. Rev. B 73, 075303 (2006).

${ }^{13}$ L. H. Ryder, Quantum Field Theory (Cambridge University Press, Cambridge, 1985), Secs. 3.5-3.6.

${ }^{14}$ G. Grosso and G. P. Parravicini, Solid State Physics (Academic, New York, 2000), Sec. 5.2.

${ }^{15}$ Ming-Hao Liu and Ching-Ray Chang, Phys. Rev. B 74, 195314 (2006).

${ }^{16}$ J. Nitta, F. E. Meijer, and H. Takayanagi, Appl. Phys. Lett. 75, 695 (1999).

${ }^{17}$ B. Molnár, F. M. Peeters, and P. Vasilopoulos, Phys. Rev. B 69, 155335 (2004). 